\title{
Train Bi-Control Problem on Riemannian Setting
}

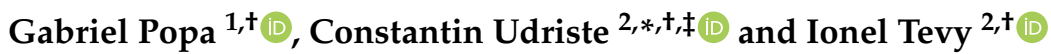 \\ 1 Department Railway Rolling Stock, Faculty of Transport, University Politehnica of Bucharest, \\ Splaiul Independentei 313, 060042 Bucharest, Romania; gabi21popa@yahoo.com \\ 2 Department of Mathematics and Informatics, Faculty of Applied Sciences, \\ University Politehnica of Bucharest, Splaiul Independentei 313, 060042 Bucharest, Romania; \\ vascatevy@yahoo.fr \\ * Correspondence: constantin.udriste@upb.ro; Tel.: +40-745-371-684 \\ + These authors contributed equally to this work. \\ $\ddagger$ Second address: Academy of Romanian Scientists, Ilfov 3, 050044 Bucharest, Romania.
}

Citation: Popa, G.; Udriste, C.; Tevy,

I. Train Bi-Control Problem on Riemannian Setting. Mathematics 2021, 9, 2898. https://doi.org/ $10.3390 /$ math 9222898

Academic Editors: Xiangmin Jiao and Marco Pedroni

Received: 8 October 2021

Accepted: 12 November 2021

Published: 14 November 2021

Publisher's Note: MDPI stays neutral with regard to jurisdictional claims in published maps and institutional affiliations.

Copyright: (c) 2021 by the authors. Licensee MDPI, Basel, Switzerland. This article is an open access article distributed under the terms and conditions of the Creative Commons Attribution (CC BY) license (https:// creativecommons.org/licenses/by/ $4.0 /)$.

\begin{abstract}
This article refers to the optimization of the energy consumption of guided traction rails, such as those used for electric trains (including subway electric units), railcars, locomotives, and trams, in a Riemannian framework. The proposed optimization strategy takes into account the compliance time drive and aims at improving the transport system for given operation conditions. Our study has five targets: (1) improving the optimal control techniques; (2) establishing a strategy for the operating conditions of the vehicle; (3) formulating and solving additional problems of optimal movement; (4) improving automatic systems for vehicle traction to optimize energy consumption in a Riemannian context; (5) formulating and solving a problem of maximizing the profit of the train. Some significant figures and formulas obtained by Maple procedures clarify the problems.
\end{abstract}

Keywords: optimal control involving ODEs; train optimal bi-control; adjoint variables; speed profile

MSC: 49K15; 53C21; 93E20

\section{Introduction}

We begin by taking into consideration the issues discussed in the reference papers: (i) the automatic train control problem [1]; (ii) optimal driving strategies for a train journey with non-zero track gradient and speed limits [2,3]; (iii) pseudospectral optimal train control [4]; (iv) energy-efficient train control [5-8]; (v) optimal train control via switched system dynamic optimization [9]; (vi) a survey on optimal trajectory planning for train operations [10]; (vii) a multiphase optimal control method for multi-train control and scheduling on railway lines [11].

The optimal control of the driving of trains has been a field of active research for many years. The optimization objective is to drive a train so that the use of energy is minimized due to time constraints and physical limitations. In this paper, we consider a more detailed nonlinear train model based on Riemannian setting and adjoint variables with two challenging, non-standard constraints: a speed constraint that is piecewiseconstant with respect to the train's position, and control constraints that are non-smooth functions of the train's speed.

We also clarify the concepts of the cost functional, ODE constraints, isoperimetric constraint, and Pontryagin maximum principle for a train control problem. The papers [12-15] introduce the possibility of passing to stochastic perturbation in the problem of optimal train control.

The original contributions of this study are as follows: Section 2 considers the Riemannian framework, which introduces a feedback control represented by the affine connection; Section 3 introduces and studies the train bi-control problem and explains the role of the adjoint variable; Section 4 describes the speed profile solving energy-efficient train movement 
by some triple of functions describing optimality (representing an accelerate-coast-brake strategy), based on the dual variable behavior; Section 5 gives significant figures obtained by Maple procedures, and Section 6 solves the problem of maximizing the profit of the train.

The research application proposed in this paper represents a possibility for the current research domain of railway transportation science. This paper continues the study begun in our papers $[15,16]$ regarding controlled problems with mathematical and engineering specifics.

Here, all our research is based on a 1-dimensional Riemannian framework.

Let $\mathbb{R}$ be the set of all real numbers and $x$ an arbitrary point of $\mathbb{R}$. A Riemannian metric on $\mathbb{R}$ is a $C^{\infty}$ function $g: \mathbb{R} \rightarrow(0, \infty)$. This metric yields the arclength $d s^{2}=g(x) d x^{2}$ and the linear connection

$$
\Gamma=\frac{1}{2} g^{-1} \frac{d g}{d x}=\frac{d}{d x} \ln \sqrt{g} .
$$

The solutions of the second order ODE

$$
\frac{d^{2} x}{d t^{2}}+\Gamma(x)\left(\frac{d x}{d t}\right)^{2}=0
$$

are the geodesics of the Riemannian manifold $(\mathbb{R}, g)$.

An interesting case is $\Gamma(x)=\frac{1}{x}$, i.e., $g(x)=x^{2}, x \neq 0$. In this case, we obtain the geodesics $x(t)=c_{1} e^{c_{2} t}$, determined by two arbitrary constants $c_{1}, c_{2}$.

The flatness $\Gamma(x)=0$ means the Euclidean context, i.e., $g(x)=1$.

If $\gamma(t)=\Gamma(x(t))=1$ throughout, then the geodesics are $x(t)=\ln \left(C_{1} t+C_{2}\right)$; for initial conditions $x(0)=1,(D(x))(0)=1$ (Maple notations), we find the geodesic $x(t)=$ $1+\ln (t+1), t>-1$.

If $\gamma(t)=\Gamma(x(t))=-1$ throughout, then the geodesics are $x(t)=-\ln \left(-C_{1} t-C_{2}\right)$; for initial conditions $x(0)=1,(D(x))(0)=-1$ (Maple notations), we obtain the geodesic $x(t)=1-\ln (t+1), t>-1$.

A model of the minimum time problem controlled by an affine connection was published recently in our paper [15].

\section{Main Requirements for Safe Railway System}

In order for rail transport to be a viable alternative for travelers and carriers, it must meet certain conditions, such as low transport time, low cost, comfort, safety, accessibility, fast links, good profit, etc. The perception of transport quality depends on a number of factors that contribute to efficient transport, both for freight transport and passenger transport. The technology enables the realization of high-performance rail vehicles. The difference between transport operators will be determined by reducing operating costs and maintenance costs. An important part of these costs are the costs of vehicles' energy consumption. Reducing these costs is a priority target for all railway companies. It is known that, between energy consumption, traffic speed, and drive time, there is a relationship of interdependence. It remains a challenge to optimize energy consumption in relation to the required time drive. The energy supplied to the vehicle traction as electric power or mechanical power (supplied by a diesel engine) is used to drive the train. Energy balance shows that part of the energy is consumed by resistances to advance (determined by the circulation on the railway profile) and the brake system. Remaining energy is found in the kinetic and potential energy. It is known that the energy consumption depends on the drive regimes selected and their sequence. It is confirmed that the optimal driving strategy for a train takes the form of a power-speed hold-coast-brake strategy, unless the track contains steep grades.

The mathematical data used in the study of train control are as follows: $T$ is the time allowed for the journey, $X$ is the distance between two stations, $u(t)$ is the acceleration applied to the train, $v(t)$ is the speed of the train, and $-r(v(t))$ is the resistive acceleration 
due to the friction. Let us accept that the movement of the train is governed by the Riemannian Newton law

$$
\ddot{x}(t)+\Gamma(x(t))(\dot{x}(t))^{2}=u(t)-r(v(t)),
$$

where $r(v), v \in[0, \infty)$ is a strictly increasing and convex function, the acceleration $u(t)$ (control variable) is limited by the relation $|u(t)| \leq 1$, and the feed-back control (pullback) $\Gamma(x(t))=\gamma(t)$ is limited also to $|\Gamma(x(t))| \leq 1$. The theory of energy consumption [7] involves also the positive part $u_{+}$of the control $u(t)$. This new function is defined by

$$
u_{+}(t)=\frac{1}{2}(u(t)+|u(t)|)
$$

The most accepted resistive force $r(v)$ is an increasing and convex trinomial

$$
r(v)=a+b v+c v^{2}, v \in[0, \infty)
$$

where $a, b, c$ are known real numbers subject to $a>0, b>0, c>0$. Generally, if $f(v)$ is a positive increasing convex function for $v \geq 0$, then

$$
f\left(\frac{1}{P_{n}} \sum_{i=1}^{n} p_{i} v_{i}\right) \leq \frac{1}{P_{n}} \sum_{i=1}^{n} p_{i} f\left(v_{i}\right),
$$

where $p_{i} \geq 0, P_{n}=\sum_{i=1}^{n} p_{i}$.

The circumstances that make train control a pressing problem at the present time are very well-known. However, automatic control cannot be achieved without knowledge of the mathematical theory of optimal control.

This article is addressed not only to mathematicians wishing to know more about the mathematical issues associated with concrete applications, but also to engineers already acquainted with classical techniques of optimal control, wishing to become more familiar with the more modern approaches of geometric control and other mathematical notions that have provided significant enhancements in classical train problems, or to experts in nonlinear control wishing to learn about the applications of this discipline to nontrivial examples in transport problems.

Solving the problem of optimizing the energy consumption of railway traction vehicles represents a priority imposed by the conditions of transport optimization and the environmental conditions. This approach requires a complex mathematical analysis of the type implemented in this paper. Efficient energy consumption requires strict control of the drive regimes in order to optimize them. This is the first step in achieving automatic control for railway vehicles.

Our aim is to review and detail the optimal control theory of train movement compared with the presentations in the papers $[1-3,9,10]$, which refer to the Euclidean setting.

\section{An Interactive Approach via Maple}

If $\gamma(t)=\Gamma(x(t))=1, u(t)=1$ throughout (right-right strategy), and $r(v):=$ $0.015+0.00003 v+0.000006 v^{2}$ (simulation data), then the ODE $\ddot{x}(t)=0.985-0.00003 \dot{x}(t)-$ $1.000006(\dot{x}(t))^{2}$ has the general solution

$$
\begin{gathered}
x(t)=-\frac{-5 \sqrt{39400236409}-15}{1000006} t+\frac{2540000}{500003} \\
\times \ln \left(\frac{250003000009}{985005910225}\left(C_{1} \exp \left(\frac{\sqrt{39400236409}}{100000} t\right)-C_{2}\right)^{2}\right) .
\end{gathered}
$$

This result imposes the optimal railway profile. The Lie symmetries of previous ODE are $[\xi=1, \eta=0],[\xi=0, \eta=1]$. 
If we need the velocity hodograph (see Figure 1 ), we add $i c s 4:=x(0)=1,(D(x))(0)=1$; sol $:=$ dsolve $(i c s 4$, ode $4, x(t)$, numeric, stiff $=$ true, range $=0 \ldots 30)$; $\operatorname{odeplot}(\operatorname{sol},[x(t), \operatorname{diff}(x(t), t)], 0 \ldots 30$, refine $=1)$;

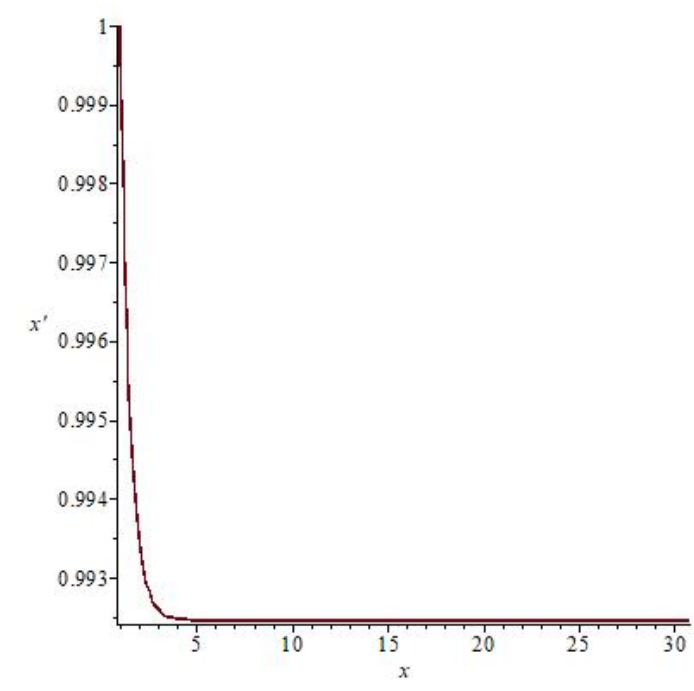

Figure 1. Velocity hodograph for moving electric train.

Similar formulas and figures can be obtained for the following three cases: $\gamma(t)=$ $\Gamma(x(t))=1, u(t)=-1$ (right-left strategy); $\gamma(t)=\Gamma(x(t))=-1, u(t)=1$ (left-right strategy); $\gamma(t)=\Gamma(x(t))=-1, u(t)=-1$ (left-left strategy).

\section{Train Bi-Control Problem}

The problem of finding the best way to drive to the next destination can be formulated as an optimal bi-control problem (local energy minimization principle). In other words, we wish to find the bi-control settings that will get the train to the next destination on time, and with minimal energy consumption.

Let us accept that Riemannian Newton's law determines the movement equation of the train. The state variables are $x$ (position) and $v$ (speed), and the control variables are $u$ and $\Gamma$.

Let us introduce the following mathematical assumptions: (i) $\mathcal{U}=L^{\infty}([0, T])$ is the set of measurable and bounded functions on the interval $[0, T]$, endowed with the supremum norm

$$
\|u\|_{\infty}=\sup _{t \in[0, T]}|u(t)|
$$

the normed space $\left(\mathcal{U},\|\cdot\|_{\infty}\right)$ is called the space of acceleration controls;

(ii) $\mathcal{W}$ is the set of measurable and bounded functions $\Gamma(x(t))=\gamma(t)$ on the interval $[0, T]$, endowed with the supremum norm (space of pullback controls);

(iii) $\mathcal{V}=C^{0,1}([0, T])$ is the set of piecewise $C^{1}$ functions $v(t)$ on the interval $[0, T]$, endowed with the norm

$$
\|v\|=\|v\|_{\infty}+\|\dot{v}\|_{\infty}
$$

A feasible triple $(u, v, \Gamma(x)) \in \mathcal{F}=\mathcal{U} \times \mathcal{V} \times \mathcal{W}$ must satisfy $\|u\|_{\infty} \leq 1, v(0)=v(T)=$ 0 , and $|\Gamma(x(t))| \leq 1$.

The isoperimetric bi-controlled train problem is: Minimize the mechanical energy consumption

$$
J(u(\cdot))=\int_{0}^{T} u_{+}(t) v(t) d t
$$


subject to

(i) two ODE constraints

$$
\dot{x}(t)=v(t), \dot{v}(t)+\Gamma(x(t)) v(t)^{2}=u(t)-r(v(t)), v(0)=v(T)=0,
$$

(ii) one isoperimetric constraint

$$
\int_{0}^{T} v(t) d t=x(T)=X
$$

(iii) two bi-control inequality constraints

$$
|u(t)| \leq 1,|\Gamma(x(t))| \leq 1
$$

If the reader is interested in unit of measures analysis, please see [4].

We seek to apply the Pontryagin maximum principle [1,10]. For this, we use the new objective functional

$$
J_{1}(u(\cdot))=\int_{0}^{T}\left(-u_{+}(t) v(t)+p_{1} v(t)\right) d t
$$

and the associated Hamiltonian

$$
H(x, u, v, \Gamma(x))=-u_{+} v+p_{1} v+p_{2}\left(-\Gamma(x(t)) v(t)^{2}+u-r(v)\right),
$$

where $p_{1}=c t$ and $p_{2}=p_{2}(t)$ are the Lagrange multipliers. The Hamiltonian can be rewritten as a piecewise function of degree at most one with respect to $u$, respectively $\Gamma(x)$. Detailing the energy consumption $u_{+}$function, we write the Hamiltonian in the form of a brace with four components:

$$
\begin{gathered}
H(x, v, u, \Gamma(x))= \\
\left\{\begin{array}{ccc}
p_{2} u+p_{1} v-p_{2}\left(\Gamma(x(t)) v(t)^{2}+r(v)\right) & \text { for } \quad-1 \leq u<0,-1 \leq \Gamma(x(t))<0 \\
p_{2} u+p_{1} v-p_{2}\left(\Gamma(x(t)) v(t)^{2}+r(v)\right) & \text { for } \quad-1 \leq u<0,0 \leq \Gamma(x(t)) \leq 1 \\
u\left(p_{2}-v\right)+p_{1} v-p_{2}\left(\Gamma(x(t)) v(t)^{2}+r(v)\right) & \text { for } \quad 0 \leq u \leq 1,-1 \leq \Gamma(x(t))<0 \\
u\left(p_{2}-v\right)+p_{1} v-p_{2}\left(\Gamma(x(t)) v(t)^{2}+r(v)\right) & \text { for } \quad 0 \leq u \leq 1,0 \leq \Gamma(x(t)) \leq 1 .
\end{array}\right.
\end{gathered}
$$

Bi-control. Since the problem is characterized by linearity both in $u$ and $\gamma$, and closed control sets, we can expect boundary solutions to occur. Indeed, if the Hamiltonian is linear in each control variable and the control variables have simple bounds, then the optimal control is a combination of bang-bang controls and singular arcs.

In our case, the Hamiltonian is piecewise linear (function of degree at most one) in each control, i.e., $u(t), \gamma(t)$, each control variable has simple bounds, and the switching functions are $p_{2}(t)$ and $p_{2}(t)-v(t)$, respectively, which cannot be simultaneously canceled, i.e., the controls $u(t)$ and $\gamma(t)$ cannot be singular simultaneously. Therefore, the optimal controls are combinations of bang-bang controls and singular arcs. The optimal controls $u^{*}(t)$ and $\Gamma^{*}(x(t))=\gamma^{*}(t)$ are discontinuous: they jump from a minimum to a maximum and vice versa in response to each change in the sign of the switching function. The case $\gamma^{*}(t)=0$ corresponds to the Euclidean setting.

(i) The two optimal controls as determined by the switching function $p_{2}(t)$ are

$$
u^{*}(t)=\left\{\begin{array}{cccc}
0 & \text { for } & p_{2}(t)>0, & \text { bang-bang control } \\
-1 & \text { for } & p_{2}(t)<0, & \text { bang-bang control } \\
? & \text { for } & p_{2}(t)=0, & \text { singular control }
\end{array}\right.
$$

respectively

$$
\gamma^{*}(t)=\left\{\begin{array}{cccc}
0 & \text { for } & p_{2}(t)>0, & \text { bang-bang control } \\
-1 & \text { for } & p_{2}(t)<0, & \text { bang-bang control } \\
? & \text { for } & p_{2}(t)=0, & \text { singular control. }
\end{array}\right.
$$


A switching time is a solution of the equation $p_{2}(t)=0$. The most interesting case is that of a finite number of switching times.

(ii) The two optimal controls as determined by the switching function $p_{2}(t)-v(t)$ are

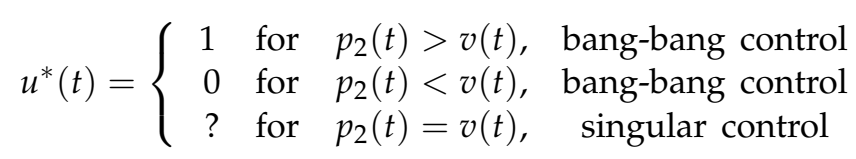

respectively

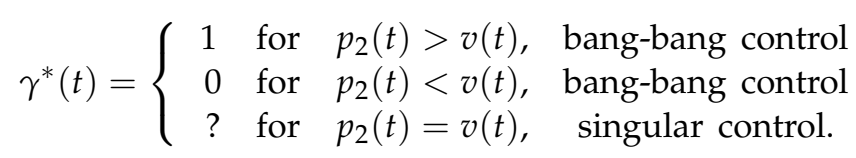

A switching time is a solution of the equation $p_{2}(t)=v(t)$. The most interesting case is that of a finite number of switching times.

State variables. The second state ODE is

$$
\dot{v}(t)=-\left(\left(c+\gamma^{*}\right) v^{2}(t)+b v(t)+a-u^{*}\right), v(t) \geq 0, v(0)=v(T)=0 .
$$

If $a-u^{*} \geq 0$, or $\Delta=b^{2}-4\left(c+\gamma^{*}\right)\left(a-u^{*}\right) \leq 0$, then $\dot{v} \leq 0$ and the speed $v$ is a decreasing function of time $t$.

If $a-u^{*}<0$, i.e., in our conditions, $u^{*}=1$ and $0<a<1$, the algebraic equation $\dot{v}(t)=0$ has two real roots $\alpha<0<\beta$ (equilibrium points). Then, the speed $v$ is an increasing function of time $t$ for $v \in[0, \beta)$ and $v$ is a decreasing function of time $t$ for $v>\beta$.

Set $u^{*}=1$. We have

$$
\frac{d v}{\left(c+\gamma^{*}\right) v^{2}+b v+a-1}=-d t
$$

or

$$
t=\frac{1}{\sqrt{\Delta_{1}}} \log C_{1}\left|\frac{v-\alpha}{v-\beta}\right|, C_{1}>0,
$$

where $\Delta_{1}=b^{2}-4\left(c+\gamma^{*}\right)(a-1)$. The condition $v(0)=0$ gives us the time

$$
t=\frac{1}{\sqrt{\Delta_{1}}} \log \left|\frac{\beta(v-\alpha)}{\alpha(v-\beta)}\right| .
$$

Remark 1. In this case, $v=\beta$ is a limit speed, so is $v \nearrow \beta$ implies $t \rightarrow \infty$.

Analogously, we obtain $t=t(v)$ in the cases $u^{*}=0$ and $u^{*}=-1$, without restriction on $v \geq 0$.

Finally, we find the integral formula (position determined by the speed)

$$
x(t)=\int_{0}^{t} v(\tau) d \tau
$$

Adjoint variable. The adjoint ODE

$$
\dot{p}_{2}(t)=-\frac{\partial H}{\partial v}
$$

becomes

$$
\dot{p}_{2}(t)=u_{+}^{*}(t)-p_{1}+p_{2}(t)\left(2 \gamma(t) v(t)+\frac{d r}{d v}(t)\right)
$$

Because

$$
\frac{d p_{2}}{d t}=\frac{d p_{2}}{d v} \frac{d v}{d t}=-\frac{d p_{2}}{d v}\left(\left(c+\gamma^{*}\right) v^{2}+b v+a-u^{*}\right),
$$


it follows the linear adjoint ODE

$$
\frac{d p_{2}}{d v}=-\frac{2\left(c+\gamma^{*}\right) v+b}{\left(c+\gamma^{*}\right) v^{2}+b v+a-u^{*}} p_{2}-\frac{u_{+}^{*}-p_{1}}{\left(c+\gamma^{*}\right) v^{2}+b v+a-u^{*}}
$$

with the general solution

$$
p_{2}(v)=\frac{\left(p_{1}-u_{+}^{*}\right) v+C_{2}}{\left(c+\gamma^{*}\right) v^{2}+b v+a-u^{*}} .
$$

\section{Speed Profile Solving Energy-Efficient Train Movement}

The authors of [7] suggested that an energy-efficient speed profile in a Euclidean setting should contain at least three or four phases coupled by continuity: (i) maximum acceleration and coast and maximum brake; (ii) maximum acceleration, hold speed, and coast and maximum brake. All the experiments confirm that these strategies are indeed efficient.

Accelerate-brake strategy. The feasible set $\mathcal{F}$ is non-empty. Indeed, the initial condition problem

$$
\dot{v}(t)=1-r(v(t)), v(0)=0
$$

has a unique solution $v_{1}(t), t \geq 0$, and the final condition problem

$$
\dot{v}(t)=-1-r(v(t)), v(T)=0
$$

has a unique solution $v_{2}(t), t \leq T$. Further, there exists a unique point $t=T_{1}$, where $v_{1}\left(T_{1}\right)=v_{2}\left(T_{1}\right)$ (the two phases are joined by continuity).

Theorem 1. The triple of functions $(u(t), \gamma(t), v(t))$, defined, respectively, by

$$
\begin{aligned}
& u(t)=\left\{\begin{array}{ccc}
1 & \text { for } & t \in\left(0, T_{1}\right) \\
-1 & \text { for } & t \in\left(T_{1}, T\right),
\end{array}\right. \\
& \gamma(t)=\left\{\begin{array}{ccc}
1 & \text { for } & t \in\left(0, T_{1}\right) \\
-1 & \text { for } & t \in\left(T_{1}, T\right),
\end{array}\right.
\end{aligned}
$$

and the continuous one,

$$
v(t)=\left\{\begin{array}{ccc}
v_{1}(t) & \text { for } & t \in\left(0, T_{1}\right) \\
v_{2}(t) & \text { for } & t \in\left(T_{1}, T\right)
\end{array}\right.
$$

satisfy the foregoing conditions and represent an accelerate-brake strategy.

Proof. Phase 1: maximum acceleration. The initial condition $v(0)=0$ implies increasing speed. This is possible for $a \in(0,1)$ only. We must take $u^{*}=1$ and $p_{2}(0)>0$. Denoting $a-1=-m^{2}$, we find $0>C_{2}=-k_{1}^{2}$. Thus, we have the time $t=\frac{1}{\sqrt{\Delta_{1}}} \log \frac{\beta(v-\alpha)}{\alpha(v-\beta)}$ and the dual variable

$$
p_{2}(v)=\frac{\left(p_{1}-1\right) v-k_{1}^{2}}{\left(c+\gamma^{*}\right) v^{2}+b v-m^{2}}
$$

Phase 2: maximum brake. The final condition $v(T)=0$ implies decreasing speed. We must take $u^{*}=-1$ and $p_{2}(T)<0$. Thus, supposing $\Delta_{-1}=b^{2}-4 c(a+1)<0$, we have

$$
T-t=\frac{2}{\sqrt{-\Delta_{-1}}} \arctan \frac{v \sqrt{-\Delta_{-1}}}{b v+2 a} \text { and } p_{2}(v)=\frac{p_{1} v-k_{2}^{2}}{\left(c+\gamma^{*}\right) v^{2}+b v+a+1} .
$$

Switch time. The equation

$$
\frac{1}{\sqrt{\Delta_{1}}} \log \frac{\beta(v-\alpha)}{\alpha(v-\beta)}+\frac{2}{\sqrt{-\Delta_{-1}}} \arctan \frac{v \sqrt{-\Delta_{-1}}}{b v+2 a}=T
$$


has a unique solution $v_{1} \in(0, \beta)$. This $v_{1}$ is the speed at which the switch breaks in and results in the corresponding time $T_{1} \in(0, T)$, necessarily.

The constants $p_{1}, k_{1}^{2}, k_{2}^{2}$ are determined by the conditions

$$
p_{1} \geq 1, \frac{\left(p_{1}-1\right) v_{1}-k_{1}^{2}}{\left(c+\gamma^{*}\right) v_{1}^{2}+b v_{1}-m^{2}}=v_{1}, \quad p_{1} v_{1}-k_{2}^{2}=0 .
$$

Unfortunately, this strategy does not fulfil the isoperimetric condition, since the speed $v$ does not depend on $p_{1}$ and $k_{1}$. Taking into account the above equation, the components $v_{1}, T$, and $X$ are connected also by the relation

$$
b T+2\left(c+\gamma^{*}\right) X=\log \frac{(a-1)\left(\left(c+\gamma^{*}\right) v_{1}^{2}+b v_{1}+a+1\right)}{(a+1)\left(\left(c+\gamma^{*}\right) v_{1}^{2}+b v_{1}+a-1\right)} .
$$

We have the next alternative. Either we give the space $X$ and calculate the time $T$, in which the space is covered, or vice versa.

Accelerate-coast-brake strategy. Let us look for more feasible pairs. With the results in the above case, for a certain $T_{2} \in\left[0, T_{1}\right]$, find the unique solution $v_{3}(t), t \geq T_{2}$ of the problem

$$
\dot{v}(t)=-r(v(t)), v\left(T_{2}\right)=v_{1}\left(T_{2}\right) .
$$

In the condition $v_{3}(T) \geq 0$, there exists a unique point $T_{3} \in\left[T_{1}, T\right]$, with $v_{3}\left(T_{3}\right)=v_{2}\left(T_{3}\right)$.

Theorem 2. The triple of functions $(u(t), \gamma(t), v(t))$, defined, respectively, by

$$
\begin{aligned}
& u(t)=\left\{\begin{array}{ccc}
1 & \text { for } & t \in\left(0, T_{2}\right) \\
0 & \text { for } & t \in\left[T_{2}, T_{3}\right] \\
-1 & \text { for } & t \in\left(T_{3}, T\right)
\end{array}\right. \\
& \gamma(t)=\left\{\begin{array}{ccc}
1 & \text { for } & t \in\left(0, T_{2}\right) \\
0 & \text { for } & t \in\left[T_{2}, T_{3}\right] \\
-1 & \text { for } & t \in\left(T_{3}, T\right)
\end{array}\right.
\end{aligned}
$$

and the continuous one,

$$
v(t)=\left\{\begin{array}{lll}
v_{1}(t) & \text { for } & t \in\left(0, T_{2}\right) \\
v_{3}(t) & \text { for } & t \in\left(T_{2}, T_{3}\right) \\
v_{2}(t) & \text { for } & t \in\left(T_{3}, T\right)
\end{array}\right.
$$

represent an accelerate-coast-brake strategy.

Proof. The optimal control $u^{*}=1$ runs while $p_{2}(v)>v$. Let us take in the previous considerations a constant $k_{1}^{2}$ such that $p_{2}\left(v_{1}\right)<v_{1}$. Because $p_{2}(0)>0=v(0)$, there exists a speed $v_{2}$ such that $p_{2}\left(v_{2}\right)=v_{2}$. This is the first positive solution of the equation

$$
v\left((c+\gamma) v^{2}+b v-m^{2}\right)=\left(p_{1}-1\right) v-k_{1}^{2} .
$$

Then,

$$
T_{2}=\frac{1}{\sqrt{\Delta_{1}}} \log \frac{\beta\left(v_{2}-\alpha\right)}{\alpha\left(v_{2}-\beta\right)}
$$

is the first switch time.

Phase 1: maximum acceleration runs as in the previous case until $t=T_{2}$. Note that both $v_{2}$ and $T_{2}$ depend upon $p_{1}$ and $k_{1}^{2}$.

Phase 2: coast. We take

$$
u^{*}=0, \dot{v}=-\left((c+\gamma) v^{2}+b v+a\right), \quad v\left(T_{2}\right)=v_{2}, \quad p_{2}(v)=\frac{p_{1} v+C_{2}}{(c+\gamma) v^{2}+b v+a} .
$$


In this situation, the condition $p_{2}\left(v_{2}\right)=v_{2}$, i.e.,

$$
\frac{p_{1} v_{2}+C_{2}}{(c+\gamma) v_{2}^{2}+b v_{2}+a}=v_{2}
$$

gives us $C_{2}=-k_{1}^{2}$ and the inequality $p_{2}(v)<v$ holds on the interval $\left(\frac{k_{1}^{2}}{p_{1}}, v_{2}\right)$ at least. Then, $T_{3}$, corresponding to $v_{3}=\frac{k_{1}^{2}}{p_{1}}$, will be the second switch time.

Phase 3: maximum brake. For $t \geq T_{3}$, we take $u^{*}=-1$ and $p_{2}(t)<0$. The evolution ODE

$$
\dot{v}(t)=-\left((c+\gamma) v^{2}(t)+b v(t)+a+1\right), \quad v\left(T_{3}\right)=v_{3}
$$

produces the dual variable

$$
p_{2}(v)=\frac{p_{1} v-k_{2}^{2}}{(c+\gamma) v^{2}+b v+a+1}
$$

where $k_{2}^{2} \geq k_{1}^{2}$. Finally, the constants $p_{1}, k_{1}^{2}, k_{2}^{2}$ are determined by the conditions

$$
p_{1} \geq 1, \quad v(T)=0, \quad \int_{0}^{T} v(t) d t=x(T)=X .
$$

\section{Significative Figures Obtained by Maple Procedures}

Today, simulation is arguably one of the most multifaceted topics encountered by a mathematician or an engineer at his/her workplace.

Maple is particularly useful for producing a graphical output in the train bi-control problem in a Riemannian setting (see Figures 2 and 3).

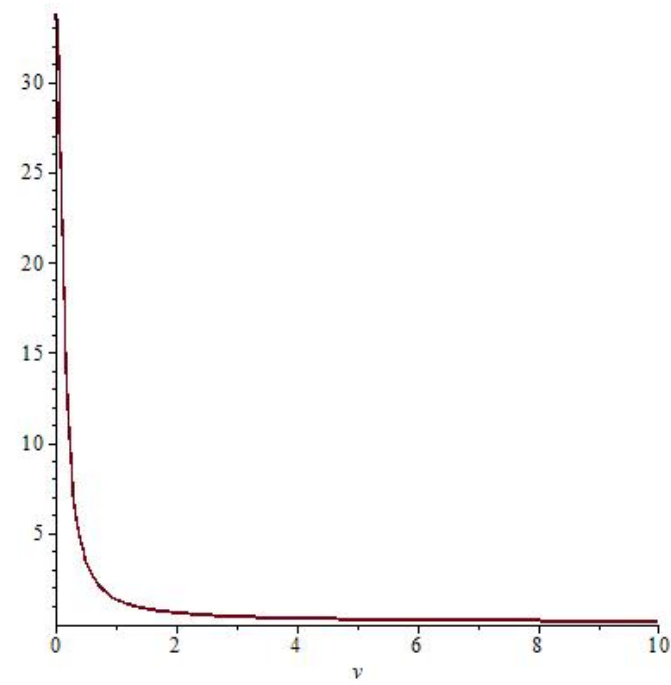

Figure 2. First adjoint variable. 


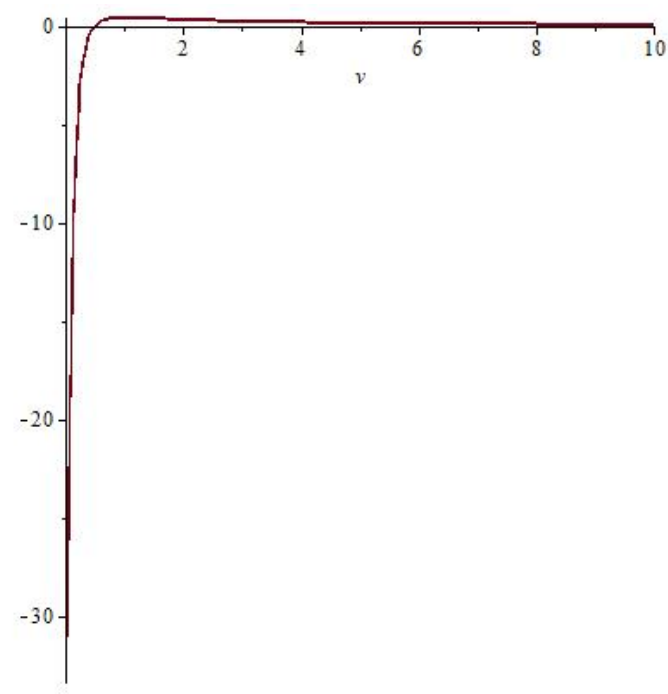

Figure 3. Second adjoint variable.

Test function (Figure 4):

$$
>r(v):=0.015+0.00003 * v+0.000006 * v^{2} ; p \operatorname{lot}(r(v), v=0 \ldots 50) ;
$$

Phase 1: maximum acceleration, first adjoint variable

$$
>p_{2}(v):=(v+0.5) /\left(1.1 * v^{2}+0.00003 * v+0.015\right) ; p \operatorname{plot}\left(p_{2}(v), v=0 \ldots 10\right)
$$

Phase 2: maximum acceleration, second adjoint variable

$$
>p_{2}(v):=(v-0.5) /\left(1.1 * v^{2}+0.00003 * v+0.015\right) ; \operatorname{plot}\left(p_{2}(v), v=0 \ldots 10\right)
$$

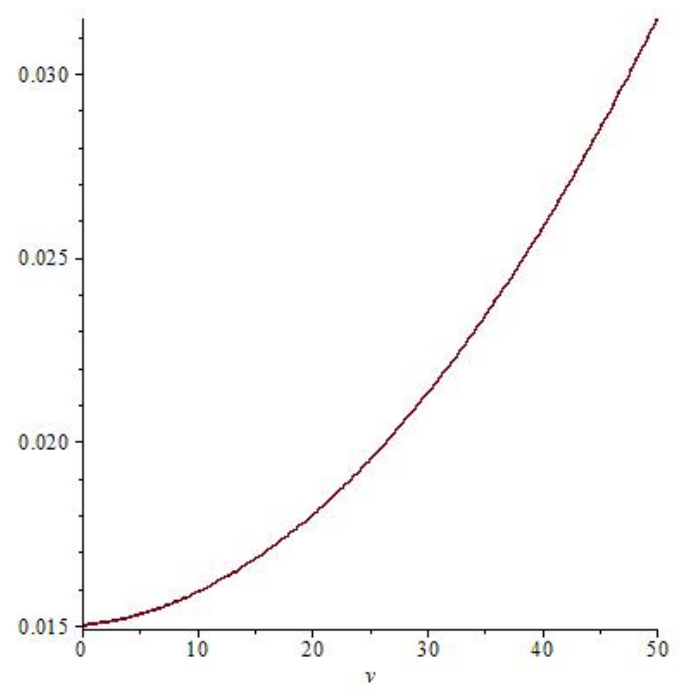

Figure 4. Increasing convex function model.

\section{Maximize the Profit of the Train}

Consider the problem of a train owner who must decide at what rate to extract profit from passenger and freight transport, at the Euclidean level, from date 0 to date $T$. At date 0 , there is $x_{0}$ profit in using the train, and the time-dependent expected amount of profit $x(t)$ declines at the rate of $u(t)$. The train owner extracts profit at cost $u(t)^{2} / x(t)$ and sells the benefit at a constant price $p$. Any profit left in use at time $T$ cannot be sold and has no 
value (there is no "scrap value"). The owner chooses the rate of impulse varying with time $u(t)$ to maximize profits over the period of ownership with no time discounting.

In the Euclidean setting, suppose that the manager wishes to maximize the profit (= revenue $-\cos$ )

$$
\Pi=\int_{0}^{T}\left[p u(t)-\frac{u(t)^{2}}{x(t)}\right] d t
$$

where the state variable $x(t)$ evolves by the ODE $\dot{x}(t)=-u(t)$. Form the Hamiltonian, and differentiate and build Pontryagin conditions

$$
\begin{gathered}
H=p u(t)-\frac{u(t)^{2}}{x(t)}-\lambda(t) u(t) \\
\frac{\partial H}{\partial u}=p-\lambda(t)-2 \frac{u(t)}{x(t)}=0 \\
\dot{\lambda}(t)=-\frac{\partial H}{\partial x}=-\left(\frac{u(t)}{x(t)}\right)^{2}
\end{gathered}
$$

As the train owner does not value the profit remaining at time $T$, one must impose $\lambda(T)=0$.

Using the above relations, it is easy to solve for ODE governing $\lambda(t)$, i.e., $\dot{\lambda}(t)=$ $-\frac{(p-\lambda(t))^{2}}{4}$, equivalent

$$
\lambda(t)=\frac{p\left(C_{1}+t\right)+4}{C_{1}+t}
$$

and the algebraic equation governing $u(t)$, i.e., $u(t)=x(t) \frac{p-\lambda(t)}{2}$. Using the initial and turn $T$ conditions, the ODE and algebraic equation can be solved, giving an important answer:

$$
x(t)=\frac{(4-p t+p T)^{2}}{(4+p T)^{2}} x_{0}, t \in[0, T],
$$

i.e., the optimal state variable is a polynomial function of the second degree. It follows the optimal control $u(t)=\frac{-2 x(t)}{C_{1}+t}$.

\section{Conclusions}

Optimal train control in a Riemannian setting involves two control parameters: one is the acceleration $u(t)$ and the second is a feedback control given by the Riemannian connection pullback $\gamma(t)=\Gamma(x(t))$. In this sense, we discuss a complex problem that uses the influence of a gravitational potential as a Riemannian metric. We follow Pontryagin's maximum principle, which gives necessary optimality conditions in the form of Hamiltonian ODEs. The analytical methods often encounter difficulties if more realistic conditions are considered that introduce complex nonlinear terms into the evolution equations and the constraints.

We seek to make the presentation as clear as possible by giving more details in smooth regular situations and referring to the known literature in more complicated cases. Moreover, we solve a profit problem formulated in a Euclidean context.

Highlights: We formulate a new model for the train bi-control problem with the Riemann-Newton law of movement. We review the state of the art and show that the important principles remain valid for this new model. We establish intuitive bounds on the optimal switching points.

Author Contributions: Conceptualization, G.P., C.U., and I.T.; methodology, all authors; writingoriginal draft preparation, G.P., C.U., and I.T.; writing-review and editing, C.U. and I.T.; validation, C.U.; funding, G.P. All authors have read and agreed to the published version of the manuscript.

Funding: This research was funded by Gabriel Popa. 
Acknowledgments: Many thanks to Emeritus, Paun Antonescu, from the Department of Mechanisms and Robot Theory, University Politehnica of Bucharest, for his wonderful guidance throughout this paper. The authors are grateful for the many helpful comments from the Mathematics reviewers. We are especially grateful to Associate Oana-Maria Pastae, "Constantin Brancusi" University of Tg-Jiu, for the English improvement of the manuscript. We would like to express our gratitude to Franco Giannessi and Antonio Maugeri for new perspective on variational analysis [17] and to thank the participants of the DGDS Conference 2021 for their surprising and encouraging discussions regarding topics in Riemannian settings.

Conflicts of Interest: The authors declare no potential conflicts of interest with respect to the research, authorship, and publication of this article.

\section{References}

1. Blake, E.J. The automatic train control problem. J. Am. Inst. Electr. Eng. 2013, 42, 27-34. [CrossRef]

2. Davydova, Y.; Cheng, J.; Howlett, P.G.; Pudney, P.J. Optimal driving strategies for a train journey with non-zero track gradient and speed limits. IMA J. Math. Appl. Bus. Ind. 1999, 10, 89-115.

3. Pudney, P.J.; Howlett, P.G. Optimal driving strategies for a train journey with speed limits. J. Aust. Math. Soc. Ser. B 1994, 36, 38-49. [CrossRef]

4. Goverdea, R.; Scheepmaker, G.; Wang, P. Pseudospectral optimal train control. Eur. J. Oper. Res. 2021, 292, 353-375. [CrossRef]

5. Howlett, P.G.; Milroy, I.; Pudney, P.J. Energy-efficient train control. Control Eng. Pract. 1994, 2, 193-200. [CrossRef]

6. Howlett, P.G. An optimal strategy for the control of a train. J. Aust. Math. Soc. Ser. B 1990, 31, 454-471. [CrossRef]

7. Howlett, P.G.; Pudney, P.J. Energy-Efficient Train Control; Advances in Industrial Control; Springer: London, UK, 1995.

8. Khmelnitsky, E. On an optimal control problem of train operation. IEEE Trans. Autom. Control 2002, 45, 1257-1266. [CrossRef]

9. Zhong, W.; Lin, Q.; Loxton, R.; Teo, K.L. Optimal train control via switched system dynamic optimization. Optim. Methods Softw. 2021, 36, 602-626. [CrossRef]

10. Wang, Y.; Ning, B.; Cao, F.; de Schutter, B.; van den Boom, T.J.J. A survey on optimal trajectory planning for train operations. In Proceedings of the 2011 IEEE International Conference on Intelligent Rail Transportation (ICIRT 2011), Beijing, China, 10-12 July 2011; pp. 589-594.

11. Ye, H.; Liu, R. A multiphase optimal control method for multi-train control and scheduling on railway lines. Transp. Res. Part B Methodol. 2016, 93, 377-393. [CrossRef]

12. Calin, O.; Udriste, C. Geometric Modeling in Probability and Statistics; Springer: Berlin/Heidelberg, Germany, 2014.

13. Calin, O.; Udriste, C.; Tevy, I. Stochastic sub-Riemannian geodesics on the Grushin distribution. Balk. J. Geom. Appl. 2014, $19,37-49$.

14. Udriste, C.; Damian, V. Simplified single-time stochastic maximum principle. Balk. J. Geom. Appl. 2011, 16, 155-173.

15. Udriste, C.; Tevy, I. Minimum time problem controlled by affine connection. Symmetry 2021, 13, 1391. sym13081391. [CrossRef]

16. Popa, G.; Udriste, C.; Tevy, I. Train control problem. Sci. Bull. Ser. A 2020, 82, 153-166.

17. Giannessi, F.; Maugeri, A. (Eds.) Variational Analysis and Applications; Springer: Berlin/Heidelberg, Germany, 2005. 